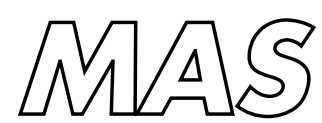

Modelling, Analysis and Simulation

\footnotetext{
Modelling, Analysis and Simulation

D. Echeverría, D. Lahaye, L. Encica, E.A. Lomonova, P.W. Hemker, A.J.A. Vandenput
} 
Centrum voor Wiskunde en Informatica (CWI) is the national research institute for Mathematics and Computer Science. It is sponsored by the Netherlands Organisation for Scientific Research (NWO).

CWI is a founding member of ERCIM, the European Research Consortium for Informatics and Mathematics.

CWI's research has a theme-oriented structure and is grouped into four clusters. Listed below are the names of the clusters and in parentheses their acronyms.

Probability, Networks and Algorithms (PNA)

Software Engineering (SEN)

\section{Modelling, Analysis and Simulation (MAS)}

Information Systems (INS)

Copyright (C) 2006, Stichting Centrum voor Wiskunde en Informatica

P.O. Box 94079, 1090 GB Amsterdam (NL)

Kruislaan 413, 1098 SJ Amsterdam (NL)

Telephone +31205929333

Telefax +31205924199

ISSN 1386-3703 


\title{
Manifold-mapping optimization applied to linear actuator design
}

\begin{abstract}
Optimization procedures in practice are based on highly accurate models that typically have an excessive computational cost. By exploiting auxiliary models that are less accurate but much cheaper to compute, space-mapping has been reported to accelerate such procedures. However, the space-mapping solution does not always coincide with the accurate model optimum. We introduce manifold mapping, an improved version of space mapping that finds this precise solution with the same computational efficiency. By an example in linear actuator design we show that our technique delivers a significant speed-up compared to other optimization schemes.
\end{abstract}

2000 Mathematics Subject Classification: 65K10, 65M60, 65N55, 65Y20, 90C31

Keywords and Phrases: space-mapping, actuators, optimization methods, surrogate-based optimization. 



\title{
Manifold-Mapping Optimization Applied to Linear Actuator Design
}

\author{
D. Echeverría ${ }^{\dagger}$, D. Lahaye ${ }^{\dagger}$, L. Encica*, E.A. Lomonova*, P.W. Hemker ${ }^{\dagger}$ and A.J.A. Vandenput* \\ $\dagger$ Centre for Mathematics and Computer Science (CWI), Kruislaan 413, 1098 SJ Amsterdam, The Netherlands \\ * Eindhoven University of Technology (TU/e), Den Dolech 2, 5600 MB Eindhoven, The Netherlands \\ \{D.Echeverria, D.Lahaye, P.W.Hemker\}@cwi.nl, \{L.Encica, E.Lomonova, A.J.A.Vandenput\}@tue.nl
}

\section{Introduction}

This paper gives full details on two design problems for a cylindrical voice-coil actuator that are introduced and solved in $[1]$.

\section{Description of the Actuator}

Linear voice-coil actuators are non-commutated electromechanical devices that provide cogging-free force outputs directly proportional to the applied current. An axisymmetrical variant consisting of a ferromagnetic core, a permanent magnet and a coil is presented in Figure 1. The ferromagnetic material is assumed to be non-linear. The $\mathrm{NdFeB}$ permanent magnet is magnetized in the vertical direction. The coil, steered by the magnetic force, moves along the $z$ axis in the gap of the core, as illustrated in Figure 2(a). The position of the coil relative to the top of the core is denoted by $D$. The minimal and maximal positions of the coil are referred to by $D=s_{\min }$ and $D=s_{\max }$, respectively. The range between $s_{\min }$ and maximal $s_{\max }$ is referred to as the stroke. Due to the axisymmetrical geometry, the force has an axial component only. It will be denoted by $F_{z}(D)$.
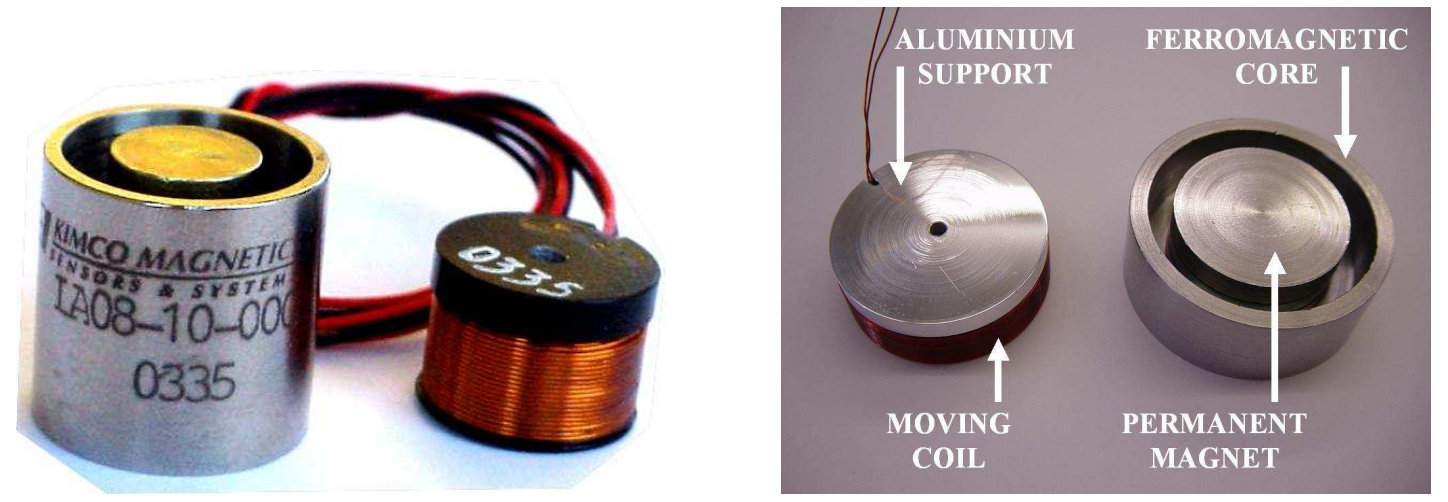

Figure 1: A cylindrical voice-coil actuator consisting of a ferromagnetic core, permanent magnet and coil.

The design variables are shown in Figure 2(b): $x_{1}$ and $x_{2}$ denote the height and radius of the magnet, $x_{3}$ and $x_{4}$ the height and thickness of the coil and $x_{5}$, and $x_{6}$ and $x_{7}$ the sizes of the core. The bounds for the design variables in the two design problems described in this report are given in Table 3 in Section 7. Two additional linear inequality constraints are imposed. The first one guarantees sufficient magnetic coupling when the coil is in its top position by imposing a lower limit on the height of the coil

$$
s_{\min }+s_{\max }+x_{6} \leq x_{3} .
$$

The second one ensures the geometry to remain feasible when the coil is in its lowest position by imposing an upper limit on the height of the coil

$$
x_{3} \leq x_{1}+x_{6}-s_{\min } .
$$

The airgap sizes $p_{1}$ and $p_{2}$ to the left and right of the coil are kept fixed. Numerical values for the parameters $s_{m i n}$, $s_{\text {max }}, p_{1}$ and $p_{2}$ used in the first and second design problems are listed in Table 4 in Section 7.

The materials properties of the actuator used in both problems can be found in the same section. 


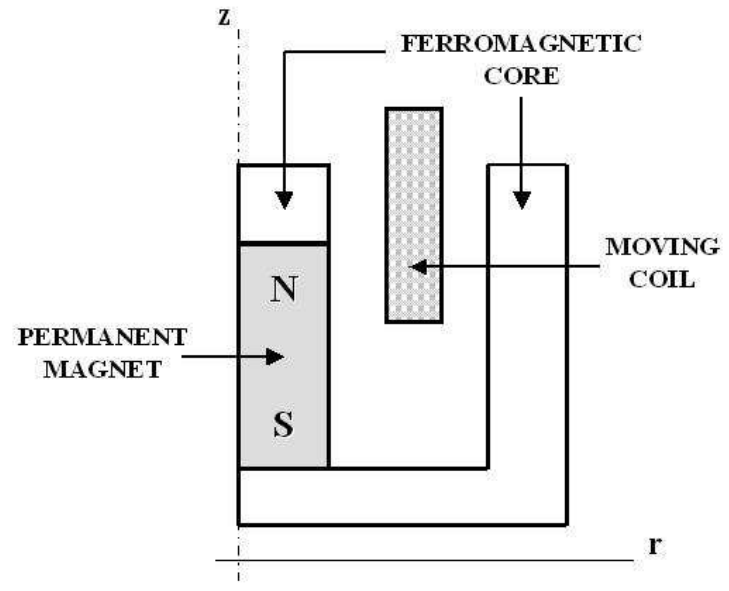

(a) Geometry.

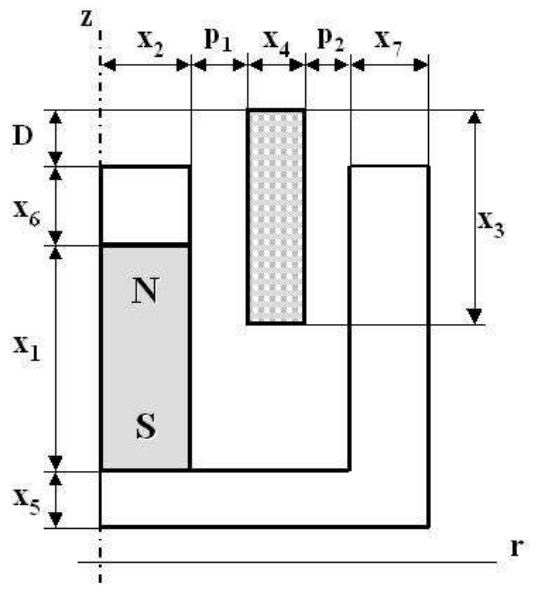

(b) Design Variables.

Figure 2: Geometry and design variables of the cylindrical voice-coil actuator.

\section{Two Optimization Problems}

\subsection{Force Response Optimization}

In the first problem we allow the coil to move over a $4 \mathrm{~mm}$ stroke, i.e., $0 \leq \mathrm{D} \leq 4 \mathrm{~mm}$. The coil movement is represented at nine equidistant points $D_{i}$ in this interval. Values for the design variables have to be found such that the force response is as flat and as close to $\mathbf{y}=24 \mathrm{~N}$ as possible. The cost function is

$$
\left[\sum_{i=1}^{9}\left[F_{z}\left(D_{i}\right)-\mathbf{y}\left(D_{i}\right)\right]^{2} / \sum_{i=1}^{9} \mathbf{y}\left(D_{i}\right)^{2}\right] \times 100 \text {. }
$$

Material parameters and variable bounds are given in Section 7.

\subsection{A More Complex Optimization Problem}

The second design problem was introduced in [2] and has non-linear equality and inequality constraints. The total mass of the actuator has to be minimized, while the mass of the coil is constrained at $10 \mathrm{~g}$. The cost function is thus the total mass of the device. The force at coil position $\mathrm{D}=4.25 \mathrm{~mm}$ should be kept at $5 \mathrm{~N}$ and the magnetic flux density in the three regions of the core indicated in Figure 3 should not exceed $1 \mathrm{~T}$. Material parameters and variable bounds are again given in Section 7.

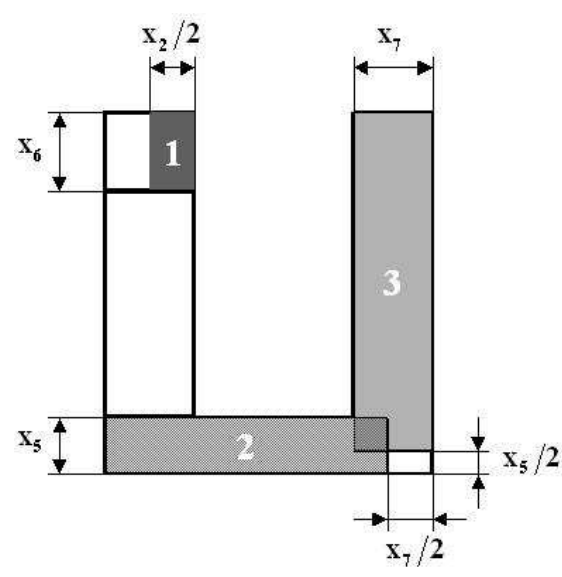

Figure 3: The average magnetic flux should not exceed $1 \mathrm{~T}$ in these three regions of the actuator core. 


\section{Space-Mapping and Manifold-Mapping}

\subsection{The Space-Mapping Technique}

Let us consider an optimization problem in the design space $X \subset \mathbb{R}^{n}$ with specifications $\mathbf{y} \in Y \subset \mathbb{R}^{m}$. The accurate behavior of electromechanical devices is often studied using models that have large computational costs, e.g., finite element models. In space-mapping (SM) terminology these models are called fine models. The fine model response is denoted by $\mathbf{f}(\mathbf{x})$, where $\mathbf{x} \in X$ is the design variable. The associated optimum is referred to as $\mathbf{x}_{f}^{*}$. SM needs a second, possibly less accurate but computationally much cheaper model. This is the coarse model; in this paper the coarse models are assumed to be defined over the same design space $X$. The coarse model response and the corresponding optimum are denoted by $\mathbf{c}(\mathbf{x})$ and $\mathbf{x}_{c}^{*} \in X$ respectively.

The SM function $\mathbf{p}: X \rightarrow X$ is defined by

$$
\mathbf{p}(\mathbf{x})=\underset{\mathbf{z} \in X}{\operatorname{argmin}}\|\mathbf{c}(\mathbf{z})-\mathbf{f}(\mathbf{x})\|
$$

SM corrects $\mathbf{x}_{c}^{*}$, the coarse optimum, in order to approximate $\mathbf{x}_{f}^{*}$. In traditional SM this is done either by solving the nonlinear system of equations,

$$
\mathbf{p}\left(\mathbf{x}_{s m_{1}}^{*}\right)=\mathbf{x}_{c}^{*},
$$

or more generally, by minimizing with the surrogate $\mathbf{c}(\mathbf{p}(\mathbf{x}))$,

$$
\mathbf{x}_{s m_{2}}^{*}=\underset{\mathbf{x} \in X}{\operatorname{argmin}}\|\mathbf{c}(\mathbf{p}(\mathbf{x}))-\mathbf{y}\|
$$

However, the solution of neither of these two problems does necessarily coincide with the fine model optimum $\mathbf{x}_{f}^{*}$.

\subsection{The Manifold-Mapping Technique}

Analyzing the conditions under which the SM solution yields the correct answer, the SM-technique can be improved in such a way that the accurate optimal design can be computed by an iterative process. Each step in this scheme requires one evaluation of the fine model $\mathbf{f}(\mathbf{x})$ and one solution of a cheap minimization problem.

Manifold-mapping (MM) replaces the SM function $\mathbf{p}(\mathbf{x})$ by an arbitrary bijection $\overline{\mathbf{p}}: X \rightarrow X$ and introduces the manifold mapping $\mathbf{S}: \mathbf{c}(X) \rightarrow \mathbf{f}(X)$. With this mapping, the point $\mathbf{c}\left(\overline{\mathbf{p}}\left(\mathbf{x}_{f}^{*}\right)\right)$ is mapped to $\mathbf{f}\left(\mathbf{x}_{f}^{*}\right)$ and the tangent space for $\mathbf{c}(X)$ at $\overline{\mathbf{p}}\left(\mathbf{x}_{f}^{*}\right)$ to the tangent space for $\mathbf{f}(X)$ at $\mathbf{x}_{f}^{*}$. By this construction then

$$
\mathbf{x}_{f}^{*}=\underset{\mathbf{x} \in X}{\operatorname{argmin}}\|\mathbf{S}(\mathbf{c}(\overline{\mathbf{p}}(\mathbf{x})))-\mathbf{y}\|
$$

In the examples in this work, the function $\overline{\mathbf{p}}(\mathbf{x})$ is taken equal to the identity.

\subsection{Implementation of Manifold-Mapping}

During the iterative solution process the manifold mapping $\mathbf{S}$ is approximated by a sequence of affine mappings $\mathbf{S}_{k}$, $k \geq 0$. This leads to the following algorithm (with $\overline{\mathbf{p}}$ an arbitrary bijection):

1. Set $k=0$ and compute $\mathbf{x}_{0}=\underset{\mathbf{x} \in X}{\operatorname{argmin}}\|\mathbf{c}(\overline{\mathbf{p}}(\mathbf{x}))-\mathbf{y}\|$.

2. Evaluate $\mathbf{f}\left(\mathbf{x}_{k}\right)$ and $\mathbf{c}\left(\overline{\mathbf{p}}\left(\mathbf{x}_{k}\right)\right)$ and finish if appropriate stopping criteria are met.

3. If $k>0$ and with $\Delta \mathbf{c}_{i}=\mathbf{c}\left(\overline{\mathbf{p}}\left(\mathbf{x}_{k-i}\right)\right)-\mathbf{c}\left(\overline{\mathbf{p}}\left(\mathbf{x}_{k}\right)\right)$ and $\Delta \mathbf{f}_{i}=\mathbf{f}\left(\mathbf{x}_{k-i}\right)-\mathbf{f}\left(\mathbf{x}_{k}\right), i=1, \cdots, \min (n, k)$, we define $\Delta C$ and $\Delta F$ to be the $m \times \min (n, k)$-matrices with respectively $\Delta \mathbf{c}_{i}$ and $\Delta \mathbf{f}_{i}$ as columns. Their singular value decompositions are respectively $\Delta C=U_{c} \Sigma_{c} V_{c}^{T}$ and $\Delta F=U_{f} \Sigma_{f} V_{f}^{T}$.

4. The approximate affine mapping is

$$
\mathbf{S}_{k} \mathbf{v}=\mathbf{f}\left(\mathbf{x}_{k}\right)+S_{k}\left(\mathbf{v}-\mathbf{c}\left(\overline{\mathbf{p}}\left(\mathbf{x}_{k}\right)\right)\right), \quad \forall \mathbf{v} \in Y,
$$

where $S_{k}$ is a regular $m \times m$ matrix equal to the identity for $k=0$ and to $\Delta F \Delta C^{\dagger}+\left(I-U_{\mathbf{f}} U_{\mathbf{f}}^{T}\right)\left(I-U_{\mathbf{c}} U_{\mathbf{c}}^{T}\right)$ if $k>0$. The ${ }^{\dagger}$ operator denotes the pseudo-inverse.

5. Compute $\mathbf{x}_{k+1}=\underset{\mathbf{x} \in X}{\operatorname{argmin}}\left\|\mathbf{S}_{k}(\mathbf{c}(\overline{\mathbf{p}}(\mathbf{x})))-\mathbf{y}\right\|$

6. Set $\mathrm{k}=\mathrm{k}+1$ and go to 2 . 


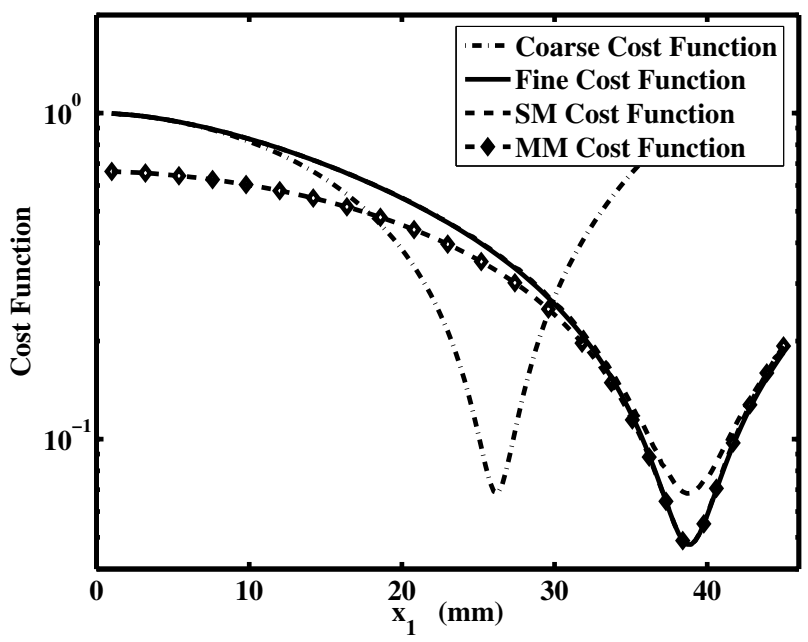

(a) One design variable.

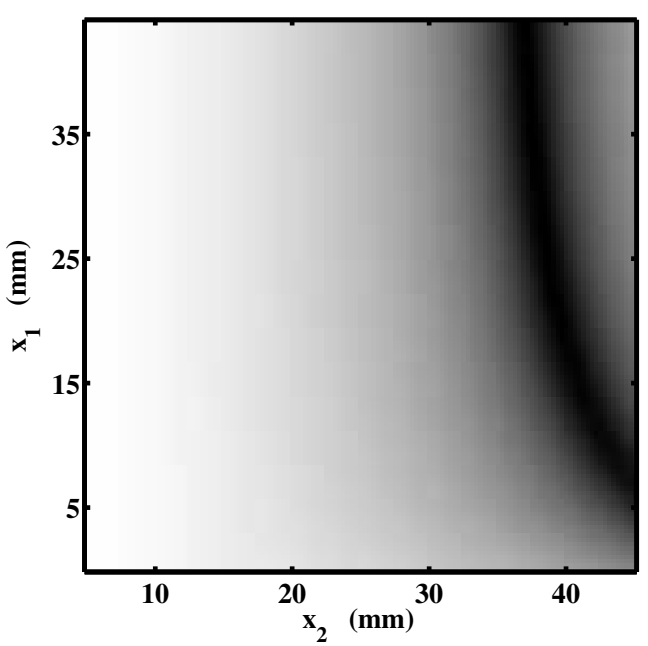

(b) Two design variables.

Figure 4: (a) Cost functions associated with the coarse (simplified FE), fine, SM and MM surrogate models for the one-dimensional problem. (b) Logarithm of the fine model cost function for the two-dimensional problem. Dark shading indicates low values for the cost function.

Step 5 can be implemented in a simpler way since it can be shown that it is asymptotically equivalent to

$$
\begin{aligned}
& \text { 5'. Compute } \mathbf{x}_{k+1}=\underset{\mathbf{x} \in X}{\operatorname{argmin}}\left\|\mathbf{c}(\overline{\mathbf{p}}(\mathbf{x}))-\overline{\mathbf{y}}_{k}\right\| \text {, where } \\
& \qquad \overline{\mathbf{y}}_{k}=\mathbf{c}\left(\overline{\mathbf{p}}\left(\mathbf{x}_{k}\right)\right)-\left[\Delta C \Delta F^{\dagger}+I-U_{c} U_{c}^{T}\right]\left(\mathbf{f}\left(\mathbf{x}_{k}\right)-\mathbf{y}\right) .
\end{aligned}
$$

The matrix $S_{k}$ satisfies

$$
S_{k}\left(\mathbf{c}\left(\overline{\mathbf{p}}\left(\mathbf{x}_{l}\right)\right)-\mathbf{c}\left(\overline{\mathbf{p}}\left(\mathbf{x}_{k}\right)\right)\right)=\mathbf{f}\left(\mathbf{x}_{l}\right)-\mathbf{f}\left(\mathbf{x}_{k}\right)
$$

for $k>0$ and $l=\max (0, k-n), \cdots, k-1$ and thus, in the limit, the desirable properties of the manifold mapping $\mathbf{S}$ are fulfilled. Under convergence of the scheme given, the limit is the fine model optimum $\mathbf{x}_{f}^{*}$.

\section{Numerical Results}

\subsection{Force Response Optimization}

For the required force level the non-linear effect of the ferromagnetic core has to be taken into account in an accurate fine model description. In the fine model the force is computed by Lorentz's formula at each of the nine coil positions. Second order Lagrangian triangular finite elements (FE) [3] are used for that purpose. The number of degrees of freedom is between 8000 and 11000, yielding three digits of accuracy in the force.

The first of the two coarse models considered is a FE model in which the non-linear material characteristic of the core is linearized. Depending on the number of Newton iterations required, this model is a factor between 30 and 50 cheaper than the fine one. The second coarse model is a magnetic equivalent circuit (MEC) [4]. This model has negligible computational cost compared to the fine model.

We initially consider a one-dimensional design problem, considering changes in the first design variable $\mathbf{x}_{1}$ only. The purpose is to illustrate how the cost function associated with MM closely approximates the fine model cost function in a region close to $\mathbf{x}_{f}^{*}$. In Fig. 4(a) the following four cost-related functions in $\mathbf{x}_{1}$ are plotted: the coarse model $\left\|\mathbf{c}\left(\mathbf{x}_{1}\right)-\mathbf{y}\right\|_{2} /\|\mathbf{y}\|_{2}$, the fine model $\left\|\mathbf{f}\left(\mathbf{x}_{1}\right)-\mathbf{y}\right\|_{2} /\|\mathbf{y}\|_{2}$, the SM $\left\|\mathbf{c}\left(\mathbf{p}\left(\mathbf{x}_{1}\right)\right)-\mathbf{y}\right\|_{2} /\|\mathbf{y}\|_{2}$ and the MM $\left\|\mathbf{S}\left(\mathbf{c}\left(\mathbf{x}_{1}\right)\right)-\mathbf{y}\right\|_{2} /\|\mathbf{y}\|_{2}$ cost functions. This figure shows that the coarse and fine model optimum are clearly different, and how MM improves the cost function associated with SM in a region close to $\mathbf{x}_{f}^{*}$.

In this one-dimensional problem, both SM and MM, using either the linear FE or the MEC as coarse model, converge in four fine model evaluations. Compared with sequential quadratic programming (SQP) [5] or the NelderMead Simplex (NMS) method [6], that are similar in performance, SM and MM deliver a speed-up of a factor between four and five. 
If we consider the two-dimensional design problem, with changes in $\mathbf{x}_{1}$ and $\mathbf{x}_{2}$ only, we can clearly illustrate that the problem is ill-conditioned. In Fig. 4(b), the cost function is plotted. In this plot the dark region shows a long and steep valley in the design space with approximately the same cost function value. This means that there is no unique solution. The value found by optimization depends on factors such as starting guess and algorithm used. Uniqueness can be restored by regularization, for example, by imposing a minimal mass constraint.

\begin{tabular}{c|cccccc} 
& $\mathbf{N M S}$ & $\mathbf{S Q P}$ & $\mathbf{S M}_{\mathrm{FE}}$ & $\mathbf{S M}_{\mathrm{MEC}}$ & $\mathbf{M M}_{\mathrm{FE}}$ & $\mathbf{M M}_{\mathrm{MEC}}$ \\
\hline \# f evals. & 24 & 31 & 9 & 6 & 9 & 4 \\
\hline cost function & 4.6 & 4.6 & 4.6 & 6.5 & 4.6 & 4.6
\end{tabular}

Table 1: Two-dimensional optimization of the voice-coil actuator.

Numerical results comparing MM with NMS, SQP and SM for the two-dimensional problem are given in Table 1. The first row in this table gives the total amount of work expressed in number of equivalent fine model evaluations. These figures are approximately proportional to the total CPU time. As starting guess for the optimization procedures we used the values obtained by optimizing the MEC model. To stabilize the convergence of MM, the LevenbergMarquardt method [5] is used. The best results in terms of computational efficiency (speed-up by a factor of six) are obtained using MM with the MEC as coarse model. The NMS and SQP algorithms are again similar in performance.

\subsection{A more complex optimization problem}

In the second design problem the two-model approach is applied in a constrained sense [7]. The performance of SM and MM is compared with that of SQP. In the fine model the constraints are evaluated by the same FE model employed in the previous subsection. In the coarse model the constraints are based on a MEC model. Each coarse model related optimization is done using SQP.

\begin{tabular}{c|c|c|c} 
& \# f evals. & total mass (g) / final design $(\mathbf{m m})$ \\
\hline SQP & 56 & $81.86 /[8.5439 .79311 .4891 .8763 .8763 .1972 .524]$ \\
\hline SM & 7 & $81.11 /[8.5009 .78611 .4501 .8833 .8383 .2002 .497]$ \\
\hline MM & 6 & $81.45 /[8.5009 .78411 .4521 .8833 .8603 .2022 .515]$
\end{tabular}

Table 2: Seven-dimensional optimization of the voice-coil actuator.

Numerical results for this problem are given in Table 2. The optimization process was stopped if the relative decrease of both the cost function and 2-norm of the design parameters was smaller than $5 \cdot 10^{-3}$, provided that the constraints were met with three digits of accuracy. SM and MM show a similar behavior: convergence is reached in seven and six fine constraints evaluations respectively. SQP converged within 56 fine constraint evaluations. Because in this problem the number of constraints is smaller than the number of design parameters, we expect the SM solution to coincide with $\mathbf{x}_{f}^{*}$. We recognize this to be the case as SQP, SM and MM yield the same optimum. But even in this situation, MM offers an additional advantage over SM. The computation of the SM function $\mathbf{p}(\mathbf{x})$ is a very delicate issue [8]. MM replaces it by the identity $\left(\overline{\mathbf{p}}=\mathbf{i d}_{X}\right)$, and in a realistic problem like this one, the efficiency results are comparable.

\section{Conclusions}

We introduced two design problems for a cylindrical voice-coil actuator. The manifold-mapping technique is an optimization method that exploits approximate model information to accelerate time-consuming design procedures. Its application on the two design problems has given evidence of the computational speed-up that this method can deliver. 


\section{Additional Tables and Figures}

\begin{tabular}{|c|c|c|}
\hline \multicolumn{3}{|c|}{ First Problem } \\
\hline \hline Design variable & Lower bound (mm) & Upper bound $(\mathrm{mm})$ \\
\hline$x_{1}$ & 1 & 45 \\
$x_{2}$ & 1 & 45 \\
$x_{3}$ & 1 & 20 \\
$x_{4}$ & 0.5 & 10 \\
$x_{5}$ & 1 & 30 \\
$x_{6}$ & 1 & 30 \\
$x_{7}$ & 1 & 30 \\
\hline
\end{tabular}

\begin{tabular}{|c|c|c|}
\hline \multicolumn{3}{|c|}{ Second Problem } \\
\hline \hline Design variable & Lower bound $(\mathrm{mm})$ & Upper bound $(\mathrm{mm})$ \\
\hline$x_{1}$ & 8.25 & 30 \\
$x_{2}$ & 1 & 30 \\
$x_{3}$ & 9.25 & 59.75 \\
$x_{4}$ & 0.35 & 30 \\
$x_{5}$ & 1 & 30 \\
$x_{6}$ & 1 & 30 \\
$x_{7}$ & 1 & 30 \\
\hline
\end{tabular}

Table 3: Lower and upper bounds for the seven design variables in the first and second design problem.

\begin{tabular}{|c|c|}
\hline \multicolumn{2}{|c|}{ First Problem } \\
\hline \hline Design parameter & Value $(\mathrm{mm})$ \\
\hline$s_{\min }$ & 0 \\
$s_{\max }$ & 4 \\
$p_{1}$ & 1 \\
$p_{2}$ & 0.5 \\
\hline
\end{tabular}

\begin{tabular}{|c|c|}
\hline \multicolumn{2}{|c|}{ Second Problem } \\
\hline \hline Design parameter & Value $(\mathrm{mm})$ \\
\hline$s_{\min }$ & 0.25 \\
$s_{\max }$ & 8.25 \\
$p_{1}$ & 0.9 \\
$p_{2}$ & 0.725 \\
\hline
\end{tabular}

Table 4: Values of $s_{\min }, s_{\max }, p_{1}$ and $p_{2}$ in the first and second design problem.

\begin{tabular}{|c|c|c|c|}
\hline \multicolumn{4}{|c|}{ First Problem } \\
\hline \hline & Coil & Ferromagnetic core & Permanent magnet \\
\hline Mass density $\left(\mathrm{kg} / \mathrm{m}^{3}\right)$ & 8933 & 7872 & 7350 \\
Current density $\left(\mathrm{A} / \mathrm{m}^{2}\right)$ & $1.505 \cdot 10^{7}$ & - & - \\
Remanent flux density $B_{r}(\mathrm{~T})$ & - & - & 1.23 \\
Relative permeability $\mu_{r}$ & 1 & nonlinear $B-H$ curve & 1.09 \\
\hline
\end{tabular}

\begin{tabular}{|c|c|c|c|}
\hline \multicolumn{4}{|c|}{ Second Problem } \\
\hline \hline & Coil & Ferromagnetic core & Permanent magnet \\
\hline Mass density $\left(\mathrm{kg} / \mathrm{m}^{3}\right)$ & 8933 & 7872 & 7350 \\
Current density $\left(\mathrm{A} / \mathrm{m}^{2}\right)$ & $1.065 \cdot 10^{7}$ & - & - \\
Remanent flux density $B_{r}(\mathrm{~T})$ & - & - & 1.21 \\
Relative permeability $\mu_{r}$ & 1 & nonlinear $B-H$ curve & 1.04 \\
\hline
\end{tabular}

Table 5: Values of the material parameters in the first and second design problem. 


\begin{tabular}{|r|r||r|r|}
\hline \multicolumn{4}{|c|}{ First Problem } \\
\hline \hline$H(\mathrm{~A} / \mathrm{m})$ & $B(\mathrm{~T})$ & $H(\mathrm{~A} / \mathrm{m})$ & $B(\mathrm{~T})$ \\
\hline 0.0 & 0.0000 & 31831.0 & 2.0250 \\
159.2 & 0.2402 & 47746.5 & 2.0850 \\
318.3 & 0.8654 & 63662.0 & 2.1300 \\
477.5 & 1.1106 & 79577.5 & 2.1650 \\
636.6 & 1.2458 & 159155.0 & 2.2800 \\
795.8 & 1.3310 & 318309.9 & 2.4850 \\
1591.5 & 1.5000 & 397887.4 & 2.5850 \\
3183.1 & 1.6000 & & \\
4774.6 & 1.6830 & & \\
6366.2 & 1.7410 & & \\
7957.7 & 1.7800 & & \\
15915.5 & 1.9050 & & \\
\hline \multicolumn{4}{|c}{} \\
\hline
\end{tabular}

\begin{tabular}{|r|r||r|r|}
\hline \multicolumn{4}{|c|}{ Second Problem } \\
\hline \hline$H(\mathrm{~A} / \mathrm{m})$ & $B(\mathrm{~T})$ & $H(\mathrm{~A} / \mathrm{m})$ & $B(\mathrm{~T})$ \\
\hline 0.0 & 0.0000 & 3183.1 & 1.5239 \\
238.7 & 0.2003 & 4774.6 & 1.6260 \\
318.3 & 0.3204 & 6366.2 & 1.6980 \\
358.1 & 0.4004 & 7957.7 & 1.7300 \\
437.7 & 0.5005 & 15915.5 & 1.8700 \\
477.5 & 0.5606 & 31831.0 & 1.9900 \\
636.6 & 0.7908 & 47746.5 & 2.0400 \\
795.8 & 0.9310 & 63662.0 & 2.0699 \\
1114.1 & 1.1014 & 79577.5 & 2.0950 \\
1273.2 & 1.2016 & 159155.0 & 2.2000 \\
1591.5 & 1.3019 & 318309.9 & 2.4000 \\
2228.2 & 1.4028 & & \\
\hline \multicolumn{3}{|c}{} \\
\hline
\end{tabular}

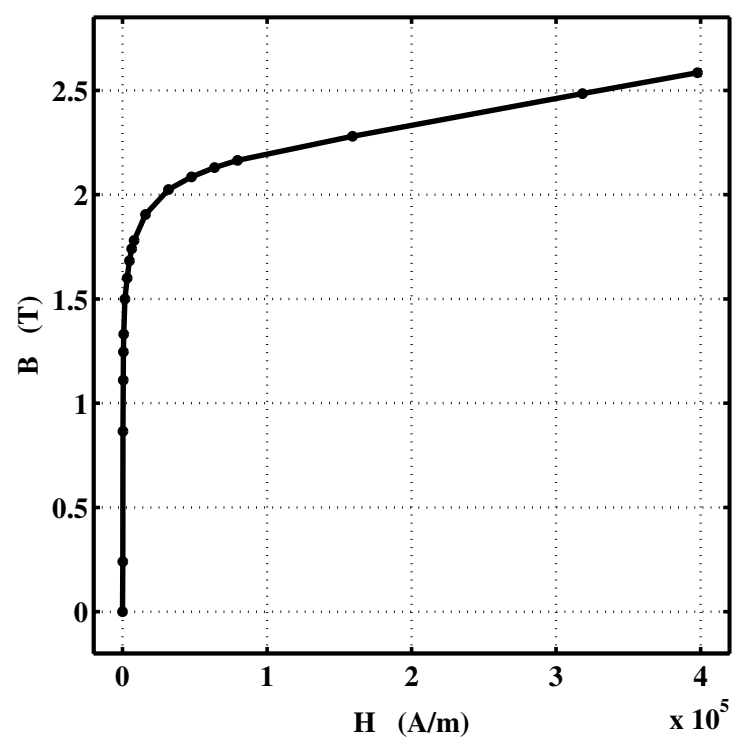

(a) First Problem

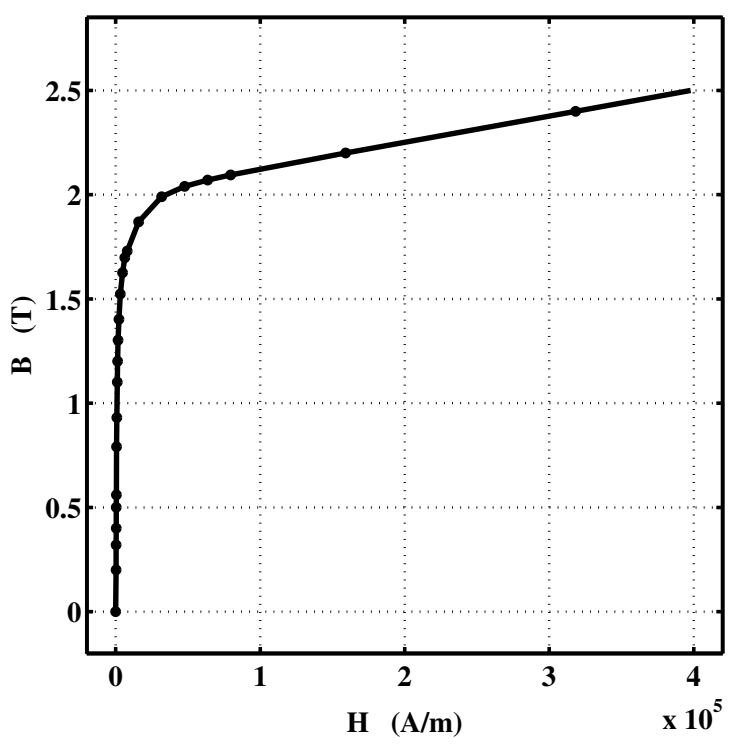

(b) Second Problem

Figure 5: $B-H$ data for the first and second design problem.

\section{Acknowledgements}

This research is supported by the Dutch Ministry of Economic Affairs within the project IOP-EMVT 02201.

\section{References}

[1] D. Echeverría, L. Lahaye, L. Encica, E.A. Lomonova, P.W. Hemker and A.J.A. Vandenput, Manifold-Mapping Optimization Applied to Linear Actuator Design, accepted, IEEE. Trans. on Magnetics, 2006.

[2] L. Encica, D. Echeverría, E. Lomonova A. J. A. Vandenput, P. W. Hemker and D. Lahaye, Efficient Optimal Design of Electromagnetic Actuators Using Space-Mapping, Sixth World Congress on Structural and Multidisciplinary Optimization (WCSMO6), Brazil, 2005.

[3] P.P Sylvester and R.L. Ferrari, Finite Element for Electrical Engineers, Cambridge University Press, Third Edition, New York, 1996.

[4] D.K. Cheng, Field and Wave Electromagnetics, Addison-Wesley, 1989. 
[5] J. Nocedal and S.J. Wright, Numerical Optimization, Springer Series in Operations Research, Springer, 1999.

[6] J. C. Lagarias, J. A. Reeds, M. H. Wright and P. E. Wright, Convergence Properties of the Nelder-Mead Simplex Method in Low Dimensions, pp. 112-147, SIAM J. Opt. 9 (1), 2003.

[7] S. J. Leary, A. Bhaskar and A. J. Keane, A Constrained Mapping Approach to the Structural Optimization of Expensive Model using Surrogates, Optimization and Engineering, pp. 385-398, 2, 2001.

[8] J. W. Bandler, Q. S. Cheng, S. A. Dakroury, A. S. Mohamed, M. H. Bakr, K. Madsen and J. Søndergaard, Space Mapping: The State of the Art, pp. 337-361, IEEE Trans. on Microwave Theory and Techniques, 52 (1), 2004. 